FULL-LENGTH REPRINT - differs from the original in layout but not in contents Bator M., Chmielewski L.J., Orłowski A., Where is the ground?

Quality measures for the planar digital terrain model in Terrestrial Laser Scanning.

In V. Murino, E. Puppo, editors, Proc. Int. Conf. ICIAP 2015, Part I, volume 9279

of Lecture Notes in Computer Science, pages 343-353, Genoa, Italy, 7-11 Sep 2015. doi:10.1007/978-3-319-23231-7_31 The copyright owner of the paper is Springer.

The final publication is available at Springer via http://dx.doi.org/10.1007/978-3-319-23231-7_31.

\title{
Where is the ground? Quality measures for the planar digital terrain model in Terrestrial Laser Scanning *
}

\author{
Marcin Bator, Leszek J Chmielewski, and Arkadiusz Orłowski \\ Faculty of Applied Informatics and Mathematics (WZIM), \\ Warsaw University of Life Sciences (SGGW), Poland \\ ul. Nowoursynowska 159, 02-775 Warsaw, Poland \\ \{marcin_bator, leszek_chmielewski, arkadiusz_orlowski\}@sggw.pl \\ http://www.wzim.sggw.pl
}

\begin{abstract}
In the analysis of terrestrial laser scanning (TLS) data the digital terrain model (DTM) is one of important elements. To evaluate the DTM or to find the DTM by way of optimization it is necessary to formulate the measure of DTM quality. Three parameterized measures are proposed and tested against a comparative model for a series of TLS data. The measure equal to the number of points inside a layer of specified height above the plane appeared to produce the most distinct maximum for an optimal model. The measures have been applied to the planar DTM but their use for other models is possible.
\end{abstract}

Keywords: digital terrain model, DTM, ground level, planar, LIDAR, TLS, quality measure, robust, optimization

\section{Introduction}

Measuring the parameters of forest regions with the LIDAR technique is gaining popularity due to constantly increasing quality of results of the methods of analysis of the data obtained. The data are in a form of the cloud of points in 3D space. Their analysis includes finding the location of the ground which is necessary as the reference level in the further computations. The ground level is modelled with the digital terrain model (DTM). The problem of finding the DTM from LIDAR data is still open (literature surveys can be found e.g. in $[1,2]$ ). Until recently, the DTMs were constructed with the use of airborne laser scanning (ALS) [3]. Works on finding the DTM from terrestrial laser scanning (TLS) data emerge recently (e.g. [4]).

In this paper the simplest DTM in the form of a single plane will be used. In our opinion the planar model of terrain is still very competitive with respect to other models due to its simplicity and sufficient accuracy in the application to forest stands measured from one LIDAR position. The measurable region extends to not more than $15-20 \mathrm{~m}$ from the LIDAR [5]. The planar model is appropriate in geographical regions where plains dominate.

\footnotetext{
* (C) Springer International Publishing Switzerland 2015 
To assess the quality of the model it is necessary to formulate a proper measure. Such a measure can be used in any optimization method. Let us consider the example of the least square error as a measure (square distance, in this case). Minimizing it leads to finding some kind of the average. In the forest data the majority of points belong to the ground, then to the tree trunks, crowns, as well as to all the other types of vegetation. The average height would encompass all these data, not only the ground points, so it would be considerably higher than the ground level due to the existence of many objects above the ground. The calculations can be further hindered by the existence of erroneous measurements. A good quality measure should operate on the data without prior selection of ground points. In this paper a number of quality measures are introduced, tested and compared.

The remainder of the paper is organized as follows. The data sets used and the problem to be solved will be described in Sect. 2. The proposed quality measures and the method of their assessment will be presented in Sect. 3 and discussed in Sect. 4. The paper will be concluded in Sect. 5 .

\section{Data}

We have used the data chosen from the TLS data sets scanned at 15 stands near Głuchów in the Grójec Forest District, Mazovian Voivodship (Central Poland), with the terrestrial LIDAR scanner FARO LS HE880, symbolically referred to as G01-G15. A data set for each stand was collected from a single position of the LIDAR scanner. The sets contained between 12 and 22 millions of measurement points belonging to the trees, other levels of vegetation like bushes and grass, and the ground.

To illustrate the further analysis we have chosen a subset of four typical examples of data. Two of them conform to the planar model very well and two others depart from it, but the distance is at an acceptable level. As the reference value for the acceptable differences in the ground location we can use the value $0.6 \mathrm{~m}$ reported in [6] as the standard deviation of the error of the digital terrain model found from ALS measurements. Such large distances were not observed in our experiments.

For the analysis we have selected a number of sectors from 3D data. A sector extends between two planes marked with dash-dotted lines in Fig. 1a and is $5^{\circ}$ wide. Such a thin sector can be easily illustrated in $2 \mathrm{D}$ with the projection onto its middle plane $V$ which forms an angle $\varphi$ with the plane $x z$. The sector extends beyond the axis $O z$ so the data for $\varphi$ and $\varphi+180^{\circ}$ are the same data, swapped around $O z$.

In this way, realistic nearly-2D data closely related to real-life data were formed. Such choice will make it possible to visualize the results conveniently for analysis, which will be explained in Sect. 3. Examples of data used and also one of those excluded from this study due to excessive terrain variation are shown in Figs. 2, 3 and in Fig. 5a. The question of choice of these data as an illustration of the presented analysis will be addressed in the following Section. 


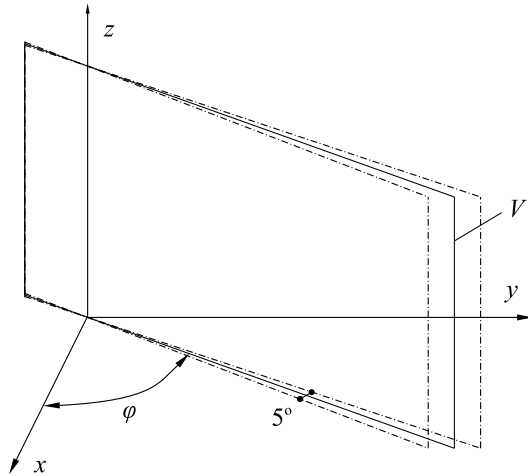

a

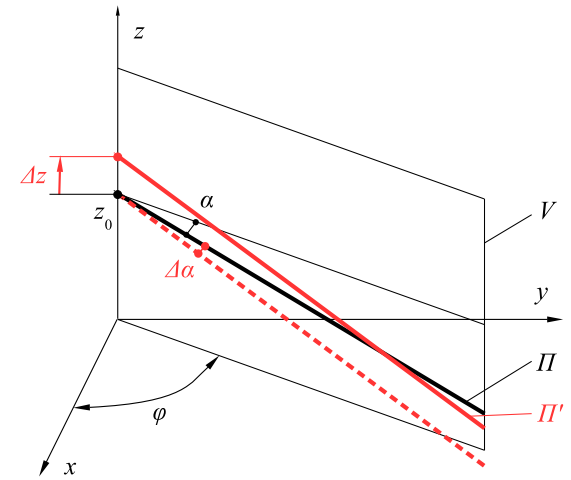

b

Fig. 1. (a) Sector from which the data are collected surrounds a plane $V$ specified by angle $\varphi$. The sector extends beyond the axis $O z$. See text in Sect. 2 for details. (b) Planar terrain model and its displacements. Displaced elements shown in red. See text in Sect. 3.2 for details.

\section{Method}

In the following we shall propose a number of quality measures for the planar terrain model. Then, these measures will be verified by examining their graphs and by comparing the model obtained by their optimization to the results calculated with the Hough transform [7]. The comparison will be made by plotting the graphs of the quality measures for a region in the parameter space surrounding the parameters of the model obtained with this comparative method. Because the data are near-2D, only one rotation and one translation of the model can be considered, so the resulting graphs will be 3D and hence will be easy to interpret. In spite of that the near-2D fragments of data are used for visualization, the comparative planes as well as the measures proposed here were calculated for the full 3D planar model (comparative model with all the points in the data set, and the proposed measures for the points belonging to the thin sector considered).

It is important to note that at this point we can not use any quantitative measure of quality of the results obtained because this is the quality measure itself that we are looking for. What is more, this measure should be used at the stage of the analysis at which the date are not yet segmented into the ground and the other classes. Therefore, the classical methods of interpolation quality assessment (cf. eg. [8]) are of little help. Due to this we shall refer to the examination of graphs which in our opinion illustrate the quality of the results in a possibly direct way. The number of graphs we can include in this paper is limited. Even in the limited set of data G01-G15 we have considered in this study the number of thin sectors is very large. From this large number of results calculated during the experiments we have tried to choose the typical ones for presentation. 


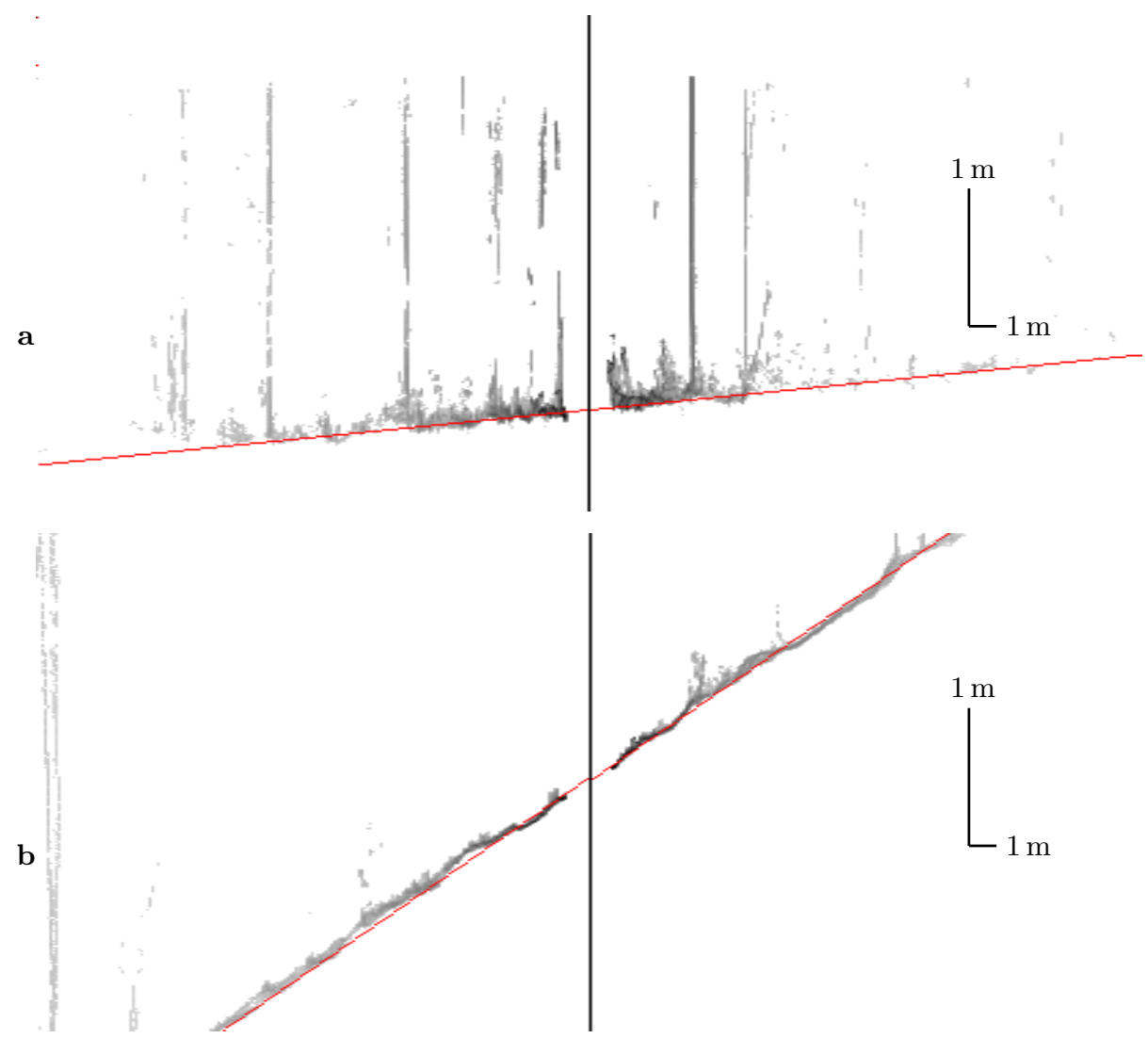

Fig. 2. Examples of good, medium, acceptably bad and excluded data (selected data sets and sectors): very good cases: (a) G01, $\varphi=95^{\circ}$, (b) G12, $\varphi=60^{\circ}$, continued in further Figs. 3 and 5 . Vertical scale is 5 times denser than the horizontal one. Maximum distance from center is $20 \mathrm{~m}$. Grey level (darkness) represents the number of measurements. Comparative ground level shown in red (see Sect. 3 for more details).

Besides the question of the measure itself, there is a need for the reference set with a credible golden standard for segmenting the ground measurement points from the other ones (trees, other vegetation, outliers and other artefacts). The reference sets reported in the literature are formed with the field surveys, photogrammetric measurements from the air, satellite radar imaging, or ALS, among others [2]. Their accuracy, especially in forest areas, is limited in comparison with that of the TLS, so new sets are needed. An interesting set which became available after this paper was first submitted is described in [9]. However, in this set the objects of interest are not the ground, but the trees, so the trees are segmented. For evaluation of results obtained for these data the criterion of visual inspection is proposed in [9], similarly as in our work. 

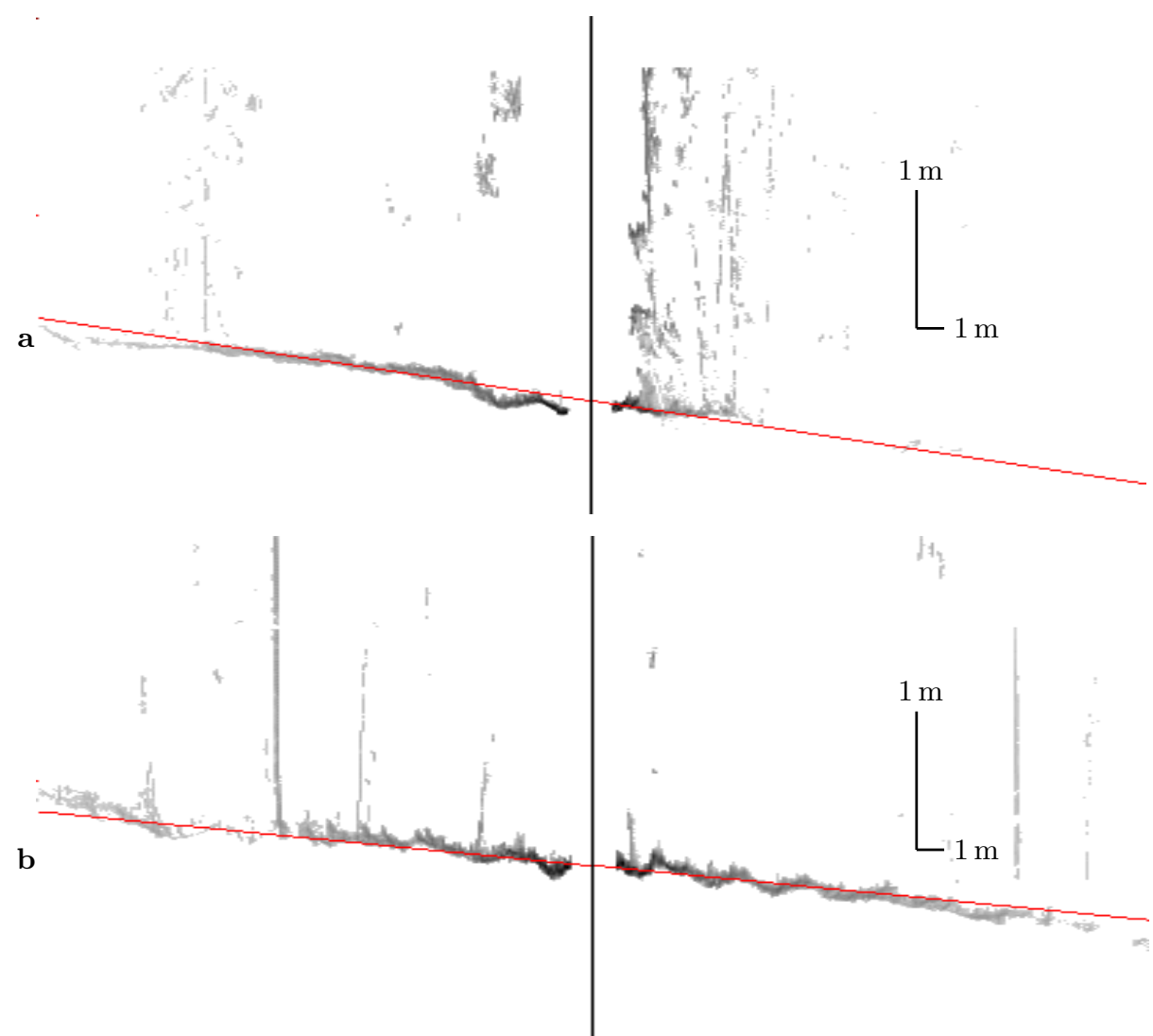

Fig. 3. Continued Fig. 2: medium cases: (a) G06, $\varphi=55^{\circ}$, (b) G15, $\varphi=20^{\circ}$.

The above considerations strongly support the need for new test data sets with reference data having appropriate accuracy for the terrestrial LIDAR measurements. However, in this study we shall resort to the data we already have.

\subsection{Quality measures proposed}

Denote an $i$-th measurement point by $P_{i}=P_{i}\left(x_{i}, y_{i}, z_{i}\right), i=1, \ldots, M$. Denote by $d\left(P_{i}, \Pi\right)$ the signed distance between this point and the planar model $\Pi$ expressed by $A x+B y+C z+D=0$. Obviously,

$$
d\left(P_{i}, \Pi\right)=\frac{A x+B y+C z+D}{\sqrt{A^{2}+B^{2}+C^{2}}} .
$$

The proposed measures will be denoted by $Q_{i}^{p}$, where $i=1, \ldots, 3$ an $\mathrm{d} p$ is a parameter. The upper index will be dropped if this does not lead to ambiguity. 
Measure $Q_{1}$ is close to the concept of the mean square error, but with a limit on distance:

$$
Q_{1}^{l}(\Pi)=\sqrt{\frac{\sum_{i=1}^{M}\left(d_{1}^{l}\left(P_{i}, \Pi\right)\right)^{2}}{\sum_{i=1}^{M} N^{l}\left(P_{i}, \Pi\right)}}
$$

where

$$
\begin{aligned}
& d_{1}^{l}\left(P_{i}, \Pi\right)= \begin{cases}d\left(P_{i}, \Pi\right) & \text { if }\left|d\left(P_{i}, \Pi\right)\right|<l, \\
0 & \text { otherwise } ;\end{cases} \\
& N^{l}\left(P_{i}, \Pi\right)= \begin{cases}1 & \text { if }\left|d\left(P_{i}, \Pi\right)\right|<l \\
0 & \text { otherwise } .\end{cases}
\end{aligned}
$$

The same measure without the limit, that is, $Q_{1}^{\infty}(\Pi)$ is the classical square error. The measure $Q_{1}$ should be minimized, leading to the least square error. As already written, the square error is not a good measure in this application, because its minimization would give the average height of all the measurement points.

Measure $Q_{2}$ is the number of measurement points inside a layer of height $l$ above and below the plane:

$$
Q_{2}^{l}(\Pi)=\sum_{i=1}^{M} N^{l}\left(P_{i}, \Pi\right) .
$$

Its maximization should yield a plane for which the neighborhood would contain a maximum number of points. The measure $Q_{2}^{\infty}$ would be simply the total number of points, which is not a reasonable measure.

Measure $Q_{3}$ is the number of measurement points inside a layer of height $l$ above the plane:

$$
\begin{gathered}
Q_{3}^{l}(\Pi)=\sum_{i=1}^{M} N_{3}^{l}\left(P_{i}, \Pi\right), \text { where } \\
N_{3}^{l}\left(P_{i}, \Pi\right)= \begin{cases}1 & \text { if } 0 \leq d\left(P_{i}, \Pi\right)<l, \\
0 & \text { otherwise } .\end{cases}
\end{gathered}
$$

Its maximization should yield a plane just under the layer containing the largest number of points.

The measure $Q_{3}^{\infty}(\Pi)$ would be the number of all points on and above the plane. It would be maximum for any plane not extending above the ground if the data contained no points under the ground, which is not the case. Practical experience shows that among the data some points which do not represent any physical objects can appear; as an example the points located extremely far from the laser, also below the ground, can be indicated. Such data points are the errors of the measurement method. The quality measures should be insensitive to them. 
In the design of the measures we have used our experience with the analysis of LIDAR data on tree stands $[10,11,12]$. However, it is apparent that the measures in which the distance limit is used can be referred to the domain of robust statistics [13]. In particular, they are close to the concept of Huber-type skipped mean, but neither of them is equivalent to it.

\subsection{Assessment}

The geometry is shown in Fig. 1b. As already written, the data points are located on the plane denoted as $V$. The intersection of the ground plane $\Pi$ with $V$ is shown with a thick black line. It is inclined versus horizon by the angle $\alpha$. Quality measures will be calculated for these data points and the plane $\Pi$ in a series of positions near its original position, denoted here by $\Pi^{\prime}$. The plane will be displaced by rotation and translation. The rotation will be made around a horizontal axis (not shown in Fig. 1b) normal to $V$ and passing through $\left(0,0, z_{0}\right)$, by an angle $\Delta \alpha$. The translation will be made vertically, by $\Delta z$. The result is a plane whose intersection with $V$ is $\Pi^{\prime}$. The rotated plane is marked with a thick dashed red line and the rotated and translated plane with a thick solid red line.

The values of $\Delta \alpha$ and $\Delta z$ were chosen so that at the practically measurable distance from the coordinate origin (15 $\mathrm{m} \mathrm{[5])} \mathrm{the} \mathrm{change} \mathrm{in} \mathrm{the} \mathrm{vertical} \mathrm{direction}$ due to rotation were up to $\pm 0.50 \mathrm{~m}$ and for translation up to $\pm 0.20 \mathrm{~m}$. As it will be seen in Sect. 4 this range contained significant maxima of the quality measures proposed and was sufficient for their assessment.

To choose the initial $(\Delta \alpha, \Delta z)=(0,0)$ position of the ground plane we have used the results from our calculations made with the Hough transform [7]. Their acceptable quality could be justified by the existence of clear maxima in the Hough space, and by visual assessment of the results (as already written, the visual inspection is unavoidable in this case).

The expected result of the present study is to find a quality measure for which a clear, global extremum of the graph $Q(\Pi)$ versus $\Delta \alpha, \Delta z$ is reached at or near the point $(0,0)$.

\section{Results and Discussion}

As the limiting distance $l$ used in Eqs. (2-6) we have used the values 0.05, 0.1 and $0.15 \mathrm{~m}$ which are close to the observed height of the layer corresponding to the ground in the data sets, as it can be seen in Figs. 2-3. This layer can be thought of as 'the grass'.

Examples of the measures $Q_{1}, Q_{2}$ and $Q_{3}$ are shown in Fig. 4. We do not show the results received for the measures $Q_{i}^{\infty}$, according to the comments on their limited applicability to the problem made in Sect. 3.1 (for example, the minimum of the square distance $Q_{1}^{\infty}$ for the data of Fig. 2a yields a plane located about $3.5 \mathrm{~m}$ above the actual ground level). 

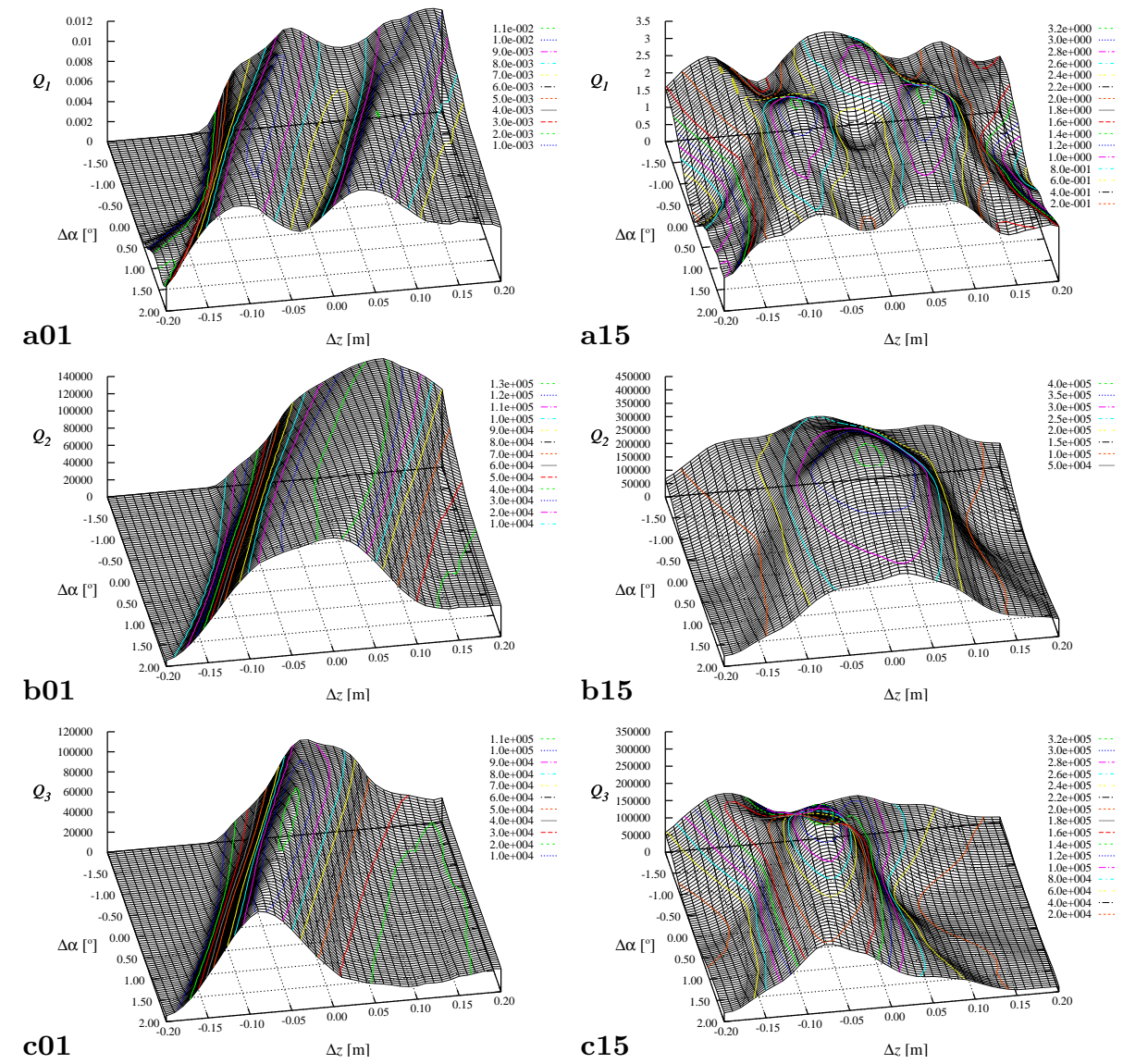

Fig. 4. Chosen quality measures for data G01 of Fig. 2a and G15 of Fig. 3b: (a) $Q_{1}$, (b) $Q_{2}$, (c) $Q_{3}$.

The shapes of the graphs of corresponding quality measures have similar shapes for all the data, so it can be attempted to draw some general conclusions from the cases shown.

The measure $Q_{1}$ has a minimum near the point $(0,0)$ in the plane $\Delta \alpha \Delta z$, but this minimum is not global and other minima can be observed in the considered region. This measure can be excluded form further considerations.

The measures $Q_{2}$ and $Q_{3}$ both have maxima near $(0,0)$ in the graphs. The maxima of $Q_{2}$ seem to be wider, but they conform with the point $(0,0)$ very well, except the case for data $G 01$. In this case the result is displaced towards positive $\Delta z$ by $0.05 \mathrm{~m}$ which is a slight difference. The maximum of the measure $Q_{3}$ is more crisp. It is displaced towards negative $\Delta z$ (downwards) by $0.05-0.07 \mathrm{~m}$, so the plane appears slightly below the reference location. This is in conformity with the assumption made to formulate Eq. (6) that the measuring points belonging to the ground are above the terrain model. 


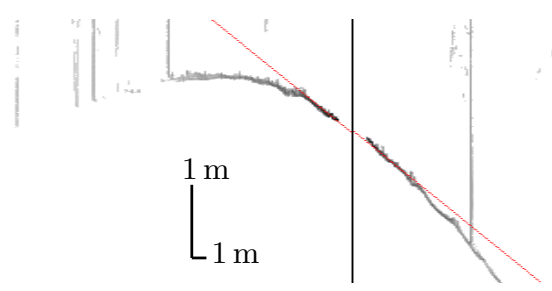

a

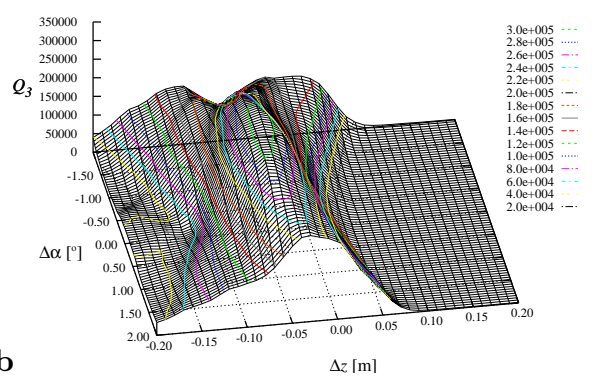

b

Fig. 5. (a) Continued Fig. 2: an example of an unacceptably bad case: G10, $\varphi=20^{\circ}$. In this case the ground level is curvilinear so it can not be approximated by a plane. (b) Quality measure $Q_{3}$ for data of Fig. a.

The differences in the vertical position obtained with $Q_{2}$ and $Q_{3}$ are small. It seems to be profitable to use the function having a more narrow maximum, so from the presented examples it follows that $Q_{3}$ can be suggested as a good solution, with $Q_{2}$ following quite immediately.

To investigate the influence of the parameter $l$ on the position of the maximum of the measures $Q_{2}$ and $Q_{3}$ we have plotted them in the $O \Delta \alpha \Delta z$ coordinates for the three used values of $l$ (Fig. 6). The measure $Q_{3}$ is stable for three from four data sets (not for G06). The measure $Q_{2}$ is stable for two from four data sets (not for G01, G06). This comparison is also in favor of $Q_{3}$.

If the traces of the plane found as a result of optimization of $Q_{3}$ were plotted in Figs. 2-3, they would be paralel to the comparative red lines, and possibly displaced down by 5 to $7 \mathrm{~cm}$, which would be less than the natural variation of heights between the ground points visible in these Figures.

Let us pay attention to the graph of $Q_{3}$ for the data G10 which can not be approximated by the planar model. In spite of this, the maximum in this graph coincides with the position of the comparative ground model. This means that the measure attains its maximum for such a position of the planar ground model which encompasses the large part of measurement points (strongly concentrated around the axis $O z$ ) and is in conformity with the model found with the compared HT method.

Finally, it should be noted that the measure $Q_{3}$, which is simply the number of points in the near vicinity above the ground model, results in a natural way from the observation that the ground is just below the most conspicuous cluster of measurement points. Such a measure is easy to implement for any DTM, irrespective of what type should it be or how many parameters should it have. Hence, it can be used in various optimization methods, including the heuristic ones. 


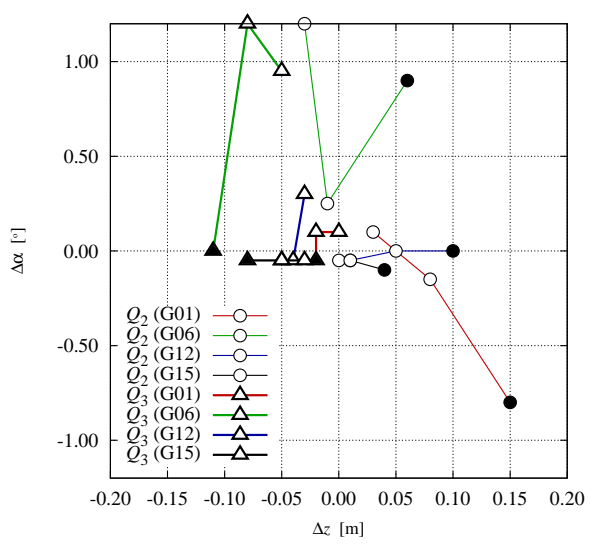

Fig. 6. Position of the minimum of measures $Q_{2}^{l}, Q_{3}^{l}$ for three values of $l$, subsequently: $0.05 \mathrm{~m}, 0.10 \mathrm{~m}, 0.15 \mathrm{~m}$. Evolution of $Q_{2}^{l}$ is marked with thin lines and circles, and that of and $Q_{3}^{l}$ with thick lines and triangles. The full symbol denotes the last used value (0.15). Results for four data sets are marked with colors: red for G01, green for G06, blue for G12 and black for G15.

\section{Conclusions}

The problem of finding the best plane which approximates the terrain, from the terrestrial laser scanning data, can be formulated as an optimization problem. As the criterion of optimization, some measures of quality proposed in this paper appeared to have the necessary properties related to the location of their maximum for the parameters of the DTM which can be considered as actually optimal. These measures are the number of points near to the terrain model, denoted as $Q_{2}^{l}$, and the number of points in the layer above the model, denoted as $Q_{3}^{l}$, which reflects the idea of locating the ground just under the layer in data in which the number of points is the largest. The parameter $l$ is related to the expected thickness of the layer. The measure $Q_{3}^{l}$ seems to perform the best in the considered application. It forms a distinct, global maximum near the optimum. The location of the maximum is relatively stable against the choice of the parameter $l$.

The considered measures have been applied and tested with the use of a simple planar terrain model but their application to other models is also possible.

There is the need for new test data sets provided with the reference data having appropriate accuracy and credibility to validate the methods applied to the analysis of the terrestrial LIDAR measurements. The acquisition of such a set and making it accessible is planned within the future research. 


\section{References}

1. Sithole, G., Vosselman, G.: Experimental comparison of filter algorithms for bare-Earth extraction from airborne laser scanning point clouds. ISPRS J. of Photogrammetry and Remote Sensing 59(1-2) (2004) 85-101 doi:10.1016/j.isprsjprs.2004.05.004.

2. Stereńczak, K., Zasada, M., Brach, M.: The accuracy assessment of DTM generated from LIDAR data for forest area - a case study for scots pine stands in Poland. Baltic Forestry 19(2) (2013) 252-262

3. Srinivasan, S., Popescu, S., Eriksson, M., Sheridan, R., Ku, N.W.: Terrestrial laser scanning as an effective tool to retrieve tree level height, crown width, and stem diameter. Remote Sensing 7(2) (2015) 1877-1896 doi:10.3390/rs70201877.

4. Puttonen, E., Krooks, A., Kaartinen, H., Kaasalainen, S.: Ground level determination in forested environment with utilization of a scanner-centered terrestrial laser scanning configuration. Geoscience and Remote Sensing Letters, IEEE 12(3) (March 2015) 616-620 doi:10.1109/LGRS.2014.2353414.

5. Zasada, M., Stereńczak, K., Dudek, W., Rybski, A.: Horizon visibility and accuracy of stocking determination on circular sample plots using automated remote measurement techniques. Forest Ecology and Management 302(0) (2013) 171-177 doi:10.1016/j.foreco.2013.03.041.

6. Stereńczak, K., Kozak, J.: Evaluation of digital terrain models generated in forest conditions from airborne laser scanning data acquired in two seasons. Scandinavian Journal of Forest Research 26(4) (2011) 374-384 doi:10.1080/02827581.2011.570781.

7. Chmielewski, L., Orłowski, A.: Ground level recovery from Terrestrial Laser Scanning data with the Variably Randomized Iterated Hierarchical Hough Transform. In: Computer Analysis of Images and Patterns: Proc. Int. Conf. CAIP 2015. Lecture Notes in Computer Science, Springer (2015) Accepted for print.

8. Kozera, R., Noakes, L.: C 1 interpolation with cumulative chord cubics. Fundamenta Informaticae 61(3-4) (August 2004) 285-301

9. Gorte, B.: Tree separation and classification contest (within the IQumulus project) (30 Apr 2015) http://homepage.tudelft.nl/41s94/iqmulus/Contest3.html. [Online; accessed 19-May-2015].

10. Chmielewski, L., Bator, M., Olejniczak, M.: Advantages of using object-specific knowledge at an early processing stage in the detection of trees in LIDAR data. Volume 8671 of Lecture Notes in Computer Science., Springer (2014) 145-154 doi:10.1007/978-3-319-11331-9_18.

11. Chmielewski, L., Bator, M.: Hough transform for opaque circles measured from outside and fuzzy voting for and against. Volume 7594 of Lecture Notes in Computer Science., Warsaw, Poland, Springer (24-26 Sep 2012) 313-320 doi:10.1007/978-3-642-33564-8_38.

12. Chmielewski, L., Bator, M., Zasada, M., Stereńczak, K., Strzeliński, P.: Fuzzy Hough transform-based methods for extraction and measurements of single trees in large-volume 3D terrestrial LIDAR data. Volume 6374 of Lecture Notes in Computer Science., Warsaw, Poland, Springer (20-22 Sep 2010) 265-274 doi : 10.1007/978-3-642-15910-7_30.

13. Huber, P.: Robust Statistics. John Wiley, New York (2003) 